Rapid Reviews COVID-19

\title{
Review 1: "SSRIs and SNRIs \\ and Risk of Death or \\ Intubation in COVID-19: \\ Results from an \\ Observational Study"
}

Sobia Haqqi

Published on: Aug 11, 2020

DOI: 10.21428/2e3983f5.bb20b43c

License: Creative Commons Attribution 4.0 International License (CC-BY 4.0). 


\section{$\underline{\text { RR:C19 Evidence Scale rating by reviewer: }}$}

- Potentially informative. The main claims made are not strongly justified by the methods and data, but may yield some insight. The results and conclusions of the study may resemble those from the hypothetical ideal study, but there is substantial room for doubt. Decision-makers should consider this evidence only with a thorough understanding of its weaknesses, alongside other evidence and theory. Decisionmakers should not consider this actionable, unless the weaknesses are clearly understood and there is other theory and evidence to further support it.

******************************************

\section{Review:}

In the present times of Covid-19, where medical management is taking precedence, the authors of this study have done a commendable job in shedding light on an equally important area of management. This is an interesting study and the findings may contribute to a broader research understanding for psychiatrists in particular and all healthcare staff in general who are the front-line warriors in these difficult times.

Study design is observational, this may limit the generalizability of this study and it is too early to comment on the Societal impact of the findings at this point in time. The conclusion drawn that all anti-depressants reduce risk of intubation or death in Covid19 patients lacks adequate support by the results. In the methodology, with respect to criteria for Sample selection, Assessment of depression at the time of initiation of antidepressant, rationale for using two anti-depressants, rationale for using more than two anti-depressants, dosages, dose adjustments, Rationale of Anti-depressant exposure to intubated patients needs further clarification.

There is no mention of Consent from the patients before treatment initiation. This can be an ethical concern. Many times in statistical analysis, the Multi-variate regression models with inverse probability are applied to minimize the effects of cofounders, which it does here but to some extent. Potential confounders such as age, sex, comorbid medical condition other than Covid-19 related, can still influence the results.

In the Results, the authors suggest that SSRI and SNRI may be associated with reduced risk of intubation or death for Covid-19. This would require further extensive research with interventional study designs, possibly multi-centred clinical trials, 
randomized and blinded, with justification of prescribing anti-depressants based on clinical assessments and valid assessment tools for diagnosing and assessing severity.

Over all, this is an interesting manuscript which is well-written, has made use of literature search and discusses limitations. 